\title{
Editorial
}

\section{Caspase-1 inflammasomes: choosing between death and taxis}

\author{
M Saleh ${ }^{1}$ and DR Green ${ }^{\star 2}$
}

\author{
Cell Death and Differentiation (2007) 14, 1559-1560. doi:10.1038/sj.cdd.4402203
}

Among the arsenal of the innate immune system are the Nodlike receptors (NLRs), which detect intracellular pathogens or other 'alarms' and participate in the formation of the inflammasome, an oligomeric platform that dimerizes and thereby activates caspase-1. The caspase, in turn, processes pro-IL1 $\beta$ and pro-IL18, key cytokines for the recruitment and engagement of inflammatory cells (hence, 'taxis'). But the activation of caspase- 1 can also trigger a form of cell death with characteristics of necrosis and apoptosis that has been called 'pyroptosis'. Two papers in this issue (FernandesAlnemri et al. ${ }^{1}$ and Petrilli et al. ${ }^{2}$ ) address the role of $\mathrm{K}^{+}$efflux in the control of caspase- 1 activation through the regulation of oligomerization of the ASC adapter molecule.

In mammals, there are approximately 20 NLRs, including NALP1b, NALP3/cryopyrin, and IPAF. Upon activation, these can oligomerize and recruit ASC, which in turn binds to caspase-1 to trigger its protease activity. Such complexes were proposed as the inflammasomes, the platforms on which caspase- 1 is activated. ${ }^{3}$

It has been known for over a decade that the activation of caspase- 1 is inhibited by $\mathrm{K}^{+}$ions, ${ }^{4}$ which efflux from the cell when the cell surface receptor P2X7 is engaged by extracellular ATP, or when microbial toxins such as nigericin, gramacidin, maitotoxin, or $\alpha$-toxin form potassium channels in the plasma membrane. Several studies had suggested that the $\mathrm{K}^{+}$efflux acts by inducing a calcium-independent phospholipase, iPLA $\mathrm{i}_{2}$, required for caspase-1 processing of cytokines. ${ }^{5}$ Now, the laboratories of Tschopp and Alnemri show that the efflux of $\mathrm{K}^{+}$directly promotes inflammasome formation by promoting the oligomerization of ASC to associate with and activate caspase-1. Petrilli et al. ${ }^{2}$ show that ASC oligomerization and recruitment of caspase- 1 by NALP3 is inhibited by $\mathrm{K}^{+}$, and provide evidence that the same may also be true for NALP1, while Fernandes-Alnemri et al. ${ }^{1}$ show that ASC can oligomerize and activate caspase-1 independent of NLRs or other proteins at sufficiently low $\mathrm{K}^{+}$ concentrations. The idea that ASC may be the target of $\mathrm{K}^{+}$regulation of caspase-1 activation is further supported by the observation that the IPAF inflammasome is not regulated by $\mathrm{K}^{+}{ }^{2}$ While ASC is required for activation of caspase-1 downstream of various inflammatory triggers, ${ }^{3}$ it is dispensable for inflammasome activity in response to Legionella, a response that requires IPAF. ${ }^{6}$ Thus, under some circumstances, a requirement for $\mathrm{K}^{+}$efflux may be overcome by engaging IPAF and bypassing ASC.

Stimuli that induce the activation of caspase- 1 to process cytokines do not necessarily lead to caspase-1-dependent cell death. What determines this choice (which we have facetiously labeled as between death and taxis)? FernandesAlnemri et al. ${ }^{1}$ suggest that the large ASC oligomer (the 'speck' that earned ASC its name: apoptosis-associated speck-like protein containing a $\mathrm{CARD}^{7}$ ), which they have dubbed the 'ASC pyroptosome', triggers pyroptosis via caspase-1. Simplistically, a low level of active caspase-1 may process cytokines without killing, as inhibitory mechanisms have sufficient time to counteract the potentially deadly effects of the caspase unless the latter exceeds some threshold. Since levels of activation do not affect the rates or specificities of proteases, different outcomes are likely to be a consequence of the thresholds set by endogenous inhibitors and counteracting mechanisms. While some inhibitors of the inflammasome are known (e.g., caspase-12, Iceberg), ${ }^{8}$ little is known about how these are engaged by different inflammasomes.

It is tempting to propose that $\mathrm{K}^{+}$levels regulate the levels of caspase-1 activation in the following way (Figure 1). When $\mathrm{K}^{+}$ levels are normal, the inflammasome is inhibited, unless IPAF is engaged. As $\mathrm{K}^{+}$levels fall, NALPs can bind to ASC and promote inflammasome formation; however, as NALPS are expressed at relatively low levels as compared to ASC and caspase-1, they limit the extent of inflammasome activity. Therefore, cytokine processing rather than death prevails. Should $\mathrm{K}^{+}$levels fall further, ASC can oligomerize independently of the NALPs, and therefore much higher levels of caspase-1 manifest to produce deadly results. It is intriguing that the activation of caspase- 1 can trigger the repair of the plasma membrane in response to microbial toxins that cause $\mathrm{K}^{+}$efflux, enhancing cell survival. ${ }^{9}$ Such feedback effects might suggest that at low levels of $\mathrm{K}^{+}$efflux, cell survival strategies persist, while at higher levels the cells trigger a caspase-1 suicide pathway.

One problem with this simple scenario, however attractive, is that in the cell-free systems employed, similar levels of $\mathrm{K}^{+}$ were inhibitory to both the NALP3 inflammasome and the ASC

1Department of Medicine, McGill University, Montreal, Quebec, Canada H3A 1 A1 and ${ }^{2}$ Department of Immunology, St. Jude Children's Research Hospital, Memphis, TN 38105, USA

*Corresponding author: DR Green, Department of Immunology, St. Jude Children's Research Hospital, Memphis, TN 38105, USA. Tel: 9014953470 ;

Fax: 901495 3107; E-mail: DGreen5240@aol.com 

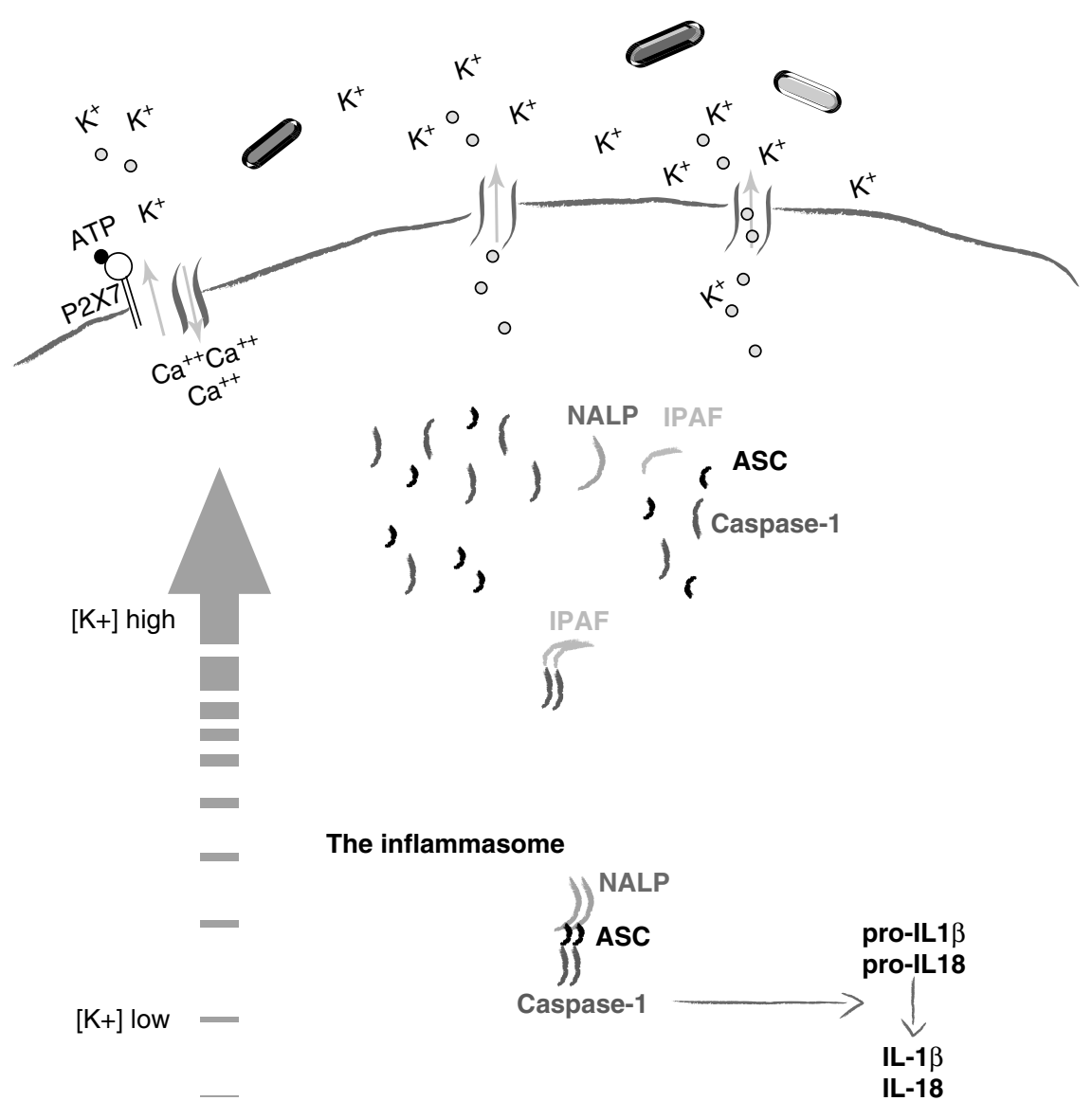

The Pyroptosome

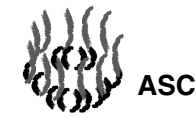

Caspase-1

Cell death ("Pyroptosis")

Burst of cytokine release

Figure 1 The control of caspase- 1 activation by $\left[\mathrm{K}^{+}\right]$levels. The cell gauges danger by monitoring its intracellular potassium levels. At normal $\left[\mathrm{K}^{+}\right]$, caspase- 1 activation is inhibited, unless IPAF is engaged. In response to inflammatory triggers and infections, potassium channels on the plasma membrane open resulting in potassium efflux, an alarm signal that induces the formation of caspase- 1 activation platforms. At intermediate $\left[\mathrm{K}^{+}\right]$concentrations, Nalps bind ASC and caspase- 1 to form the inflammasome, in which caspase- 1 is activated transiently, leading to controlled inflammation. At sufficiently low $\left[\mathrm{K}^{+}\right]$levels, ASC binds caspase-1 directly and forms the pyroptosome, in which caspase- 1 is fully activated leading to pyroptosis and a burst of cytokine release

pyroptosome. ${ }^{1,2} \mathrm{~A}$ more careful and direct comparison of the effects of $\mathrm{K}^{+}$on caspase- 1 activation under each condition may help to resolve this issue. It may also be that in cells, other factors come into play that are not faithfully mimicked in the cell-free systems.

Is this choice to die or not real? Or is it simply that at some point the need for more caspase-1 activation overwhelms the cell without performing a physiologic function? Until we can specifically regulate the cytokine processing versus cell death activities of caspase-1, the answer will remain speculative. Cell death is an effective way to remove infected cells, and it is possible that other innate mechanisms were essentially grafted to this response over evolutionary time. Through duplication and divergence of the recognition and adapter molecules, cell death versus infection-resistance mechanisms came under separate regulation. One might even wonder if apoptosis, per se, began as a response to changes in an intracellular organelle that resembles a microbe (i.e., the mitochondria). The efflux of $\mathrm{K}^{+}$, which may regulate the life and death decisions of the inflammasome, also appears to regulate the oligomerization and function of the APAF-1 apoptosome in the mitochondrial pathway of apoptosis. ${ }^{10}$

1. Fernandes-Alnemri T, Wu J, Yu J, Datta P, Miller B et al. Cell Death Differ 2007; 14: 15901604

2. Petrilli V, Papin S, Dostert C et al. Cell Death Differ 2007; 14: 1583-1589.

3. Mariathasan S, Monack DM. Nat Rev Immunol 2007; 7: 31-40.

4. Walev I, Reske K, Palmer M et al. EMBO J 1995; 14: 1607-1614

5. Walev I, Klein J, Husmann M et al. J Immunol 2000; 164: 5120-5124.

6. Zamboni DS, Kobayashi KS, Kohlsdorf T et al. Nat Immunol 2006; 7: 318-325.

7. Masumoto J, Taniguchi S, Ayukawa K et al. J Biol Chem 1999; 274: 33835-33838.

8. Scott AM, Saleh M. Cell Death Differ 2007; 14: 23-31.

9. Gurcel L, Abrami L, Girardin S. Cell 2006; 126: 1135-1145.

10. Cain K, Langlais C, Sun XM et al. J Biol Chem 2001; 276: 41985-41990. 